\title{
Extreme intensity pulses in a semiconductor laser with a short external cavity
}

\author{
Jose A. Reinoso* \\ Departamento de Fisica Fundamental, Universidad Nacional de Educacion a Distancia (UNED), \\ Paseo Senda del Rey 9, E-28040 Madrid, Spain \\ Jordi Zamora-Munt ${ }^{\dagger}$ \\ IFISC (CSIC-UIB), Campus Universitat Illes Balears, E-07122 Palma de Mallorca, Spain \\ Cristina Masoller \\ Departament de Fisica i Enginyeria Nuclear, Universitat Politecnica de Catalunya, Colom 11, ES-08222 Terrassa, Barcelona, Spain
}

(Received 14 February 2013; revised manuscript received 22 April 2013; published 19 June 2013)

\begin{abstract}
We present a numerical study of the pulses displayed by a semiconductor laser with optical feedback in the short-cavity regime, such that the external cavity round-trip time is shorter than the laser relaxation oscillation period. For certain parameters there are occasional pulses, which are high enough to be considered extreme events. We characterize the bifurcation scenario that gives rise to such extreme pulses and study the influence of noise. We demonstrate intermittency when the extreme pulses appear and hysteresis when the attractor that sustains these pulses is destroyed. We also show that this scenario is robust under the inclusion of noise.
\end{abstract}

DOI: 10.1103/PhysRevE.87.062913

PACS number(s): $05.45 .-\mathrm{a}, 42.60 . \mathrm{Mi}, 42.55 . \mathrm{Px}, 42.65 . \mathrm{Sf}$

\section{INTRODUCTION}

The study of extreme and rare events is a highly active and interdisciplinary research field [1-3]. Extreme events can have catastrophic consequences in fields such as climatology, population dynamics, and economy [4-6]. In lasers, for example, extreme and rare pulses have been observed in mode-locked lasers [7] and in semiconductor lasers with continuous-wave optical injection [8,9] or with phase-conjugated feedback [10].

We present a numerical study of the intensity pulses displayed by a semiconductor laser with optical feedback in the short-cavity regime [11-13], such that the external cavity round-trip time is shorter than the laser relaxation oscillation period. We use as a framework the well-known Lang-Kobayashi (LK) model [14-16]. Previous numerical work based on the LK model has found high-intensity pulses in the laser chaotic output, which correspond to transitions between external cavity modes (ECMs) [12]. We characterize these pulses and show that in specific parameter regions they are high enough to be considered extreme events.

In extreme value analysis the definition of an extreme event is arbitrary, as is associated with an event that is rare and that has an extreme deviation from the average. Qualitatively, extreme values are those in the tail of a long-tailed distribution; quantitatively, there are two main approaches to define extreme values: (i) values that exceed (or fall below) a certain threshold are considered extreme [17], and (ii) maxima (or minima) in "blocks" of the time series are considered extreme [18]. In this work we use the first criterion and define extreme intensity pulses as those above a certain threshold. One should note that both approaches involve a certain degree of arbitrariness, either in the selection of the threshold or in the selection of the length of the block. An example of the use of the first criterion

\footnotetext{
*jmaparicio@bec.uned.es

†jordi@ifisc.uib-csic.es

‡cristina.masoller@upc.edu
}

is in oceanography, where extreme waves (referred to as freak or rogue waves) are those whose height is larger than the mean value plus four to eight times the standard deviation of the height distribution or waves with an abnormality index larger than 2 [17]. An example of the use of the second criterion is in climate data analysis, where extreme values can be annual, biannual, etc.

Using the point-over-threshold criterion to define extreme intensity pulses, we study how they develop and how they are affected by noise. We demonstrate that an abrupt expansion in phase space of an attractor developed from an ECM creates an expanded attractor that sustains extreme pulses. For certain parameters this attractor coexists with a smaller attractor that develops from a different ECM. We identify two phenomena involved in the appearance and in the destruction of the attractor that sustains extreme pulses: deterministic intermittency when the attractor abruptly expands and hysteresis when the attractor is destroyed. We also show that this scenario is robust under the inclusion of noise.

This paper is organized as follows. Section II briefly describes the model employed, which is the well-known delay-differential LK model [14]. Section III presents the numerical results; we first focus on deterministic simulations and then discuss the influence of noise. Section IV presents a summary of the results and the conclusions.

\section{MODEL}

The rate equations for the complex optical field, $E$, and the excess carrier number, $N$, are [14]

$$
\begin{gathered}
d E / d s=(1+i \alpha) N E(s)+\eta e^{-i w \theta} E(s-\theta)+\beta \xi, \\
T d N / d s=J-N-(1+2 N)|E(s)|^{2} .
\end{gathered}
$$

In these equations the dimensionless time, $s$, and the delay time, $\theta$, are in units of photon lifetime $\tau_{p}: s=t / \tau_{p}, \theta=\tau / \tau_{p}$. The parameters are $T=\tau_{n} / \tau_{p}$, where $\tau_{n}$ is the carrier lifetime, the feedback rate is $\eta$, the feedback phase is $w \theta$, the pump 
current parameter is $J$, and the line-width enhancement factor is $\alpha$. Spontaneous emission noise is taken into account by a complex additive Gaussian white noise, $\xi$, and the noise strength is $\beta$.

The time-delayed feedback renders the system multistable and the model has several fixed-point solutions, usually referred to as ECMs, which can be calculated from

$$
\begin{gathered}
\Delta \phi_{s}=-\eta \theta \sqrt{1+\alpha^{2}} \sin \left(\Delta \phi_{s}+w \theta+\arctan (\alpha)\right), \\
E_{0 s}^{2}=\frac{J-N_{s}}{1+2 N_{s}}, \\
N_{s}=-\eta \cos \left(\Delta \phi_{s}+w \theta\right),
\end{gathered}
$$

where $\Delta \phi_{s}, E_{0 s}$, and $N_{s}$ are the steady-state values of the phase difference, field amplitude, and carrier number. The number of ECMs increases with the feedback strength, and their stability depends on the model parameters.

As $\eta$ increases new ECMs appear in pairs after saddle-node bifurcations. The initially stable ECMs lead a chaotic attractor after a series of bifurcations. With a further increase in the feedback the chaotic attractors expand and eventually merge with previously existing attactors, forming either an attractor ruin or a stable attractor [19-21]. In the first case the chaotic dynamics is a transient after which the trajectory finds a stable ECM; in the second case the attractor merging process results in a single stable attractor that either can coexist with a stable ECM or can be the only stable attractor (this occurs when there are no stable ECMs). When the chaotic dynamics is transient, it has been shown that the average duration of the transient increases several orders of magnitude for each unit increase in the value of the $\alpha$ parameter [22,23]. Here we use the same parameters as in Ref. [12], and the value of $\alpha$ is high enough to guarantee that the duration of the chaotic transient (if any) is, in practice, infinite.

\section{RESULTS}

We chose parameters similar to those in Ref. [12], where the bifurcations of the ECMs were studied in detail: $T=1710$, $J=1.155, \alpha=5$, and $\theta=70$. In the simulations the feedback strength, the feedback phase, and the noise strength are taken as control parameters. The equations are integrated with a second-order Runge-Kutta method with integration step $d s=$ 0.01 . The initial conditions are such that the complex field and carrier number are close to 0 . In the following, first we present the results of deterministic simulations and then we discuss the influence of noise.

\section{A. Deterministic simulations}

When the feedback strength varies the laser intensity displays a complicated sequence of bifurcations. In Fig. 1 we plot the amplitude of the intensity pulses and the color code indicates, on a logarithmic scale, the number of pulses, for increasing [Fig. 1(a)] and for decreasing [Fig. 1(b)] feedback. The bifurcation scenario is as discussed in [12], and a similar one has been observed with optoelectronic feedback [24]. When the feedback increases, if $\eta<0.064$, the intensity pulses are relatively small $(<4)$; however, slightly above this feedback level a sudden abrupt increase in the pulse amplitude occurs,

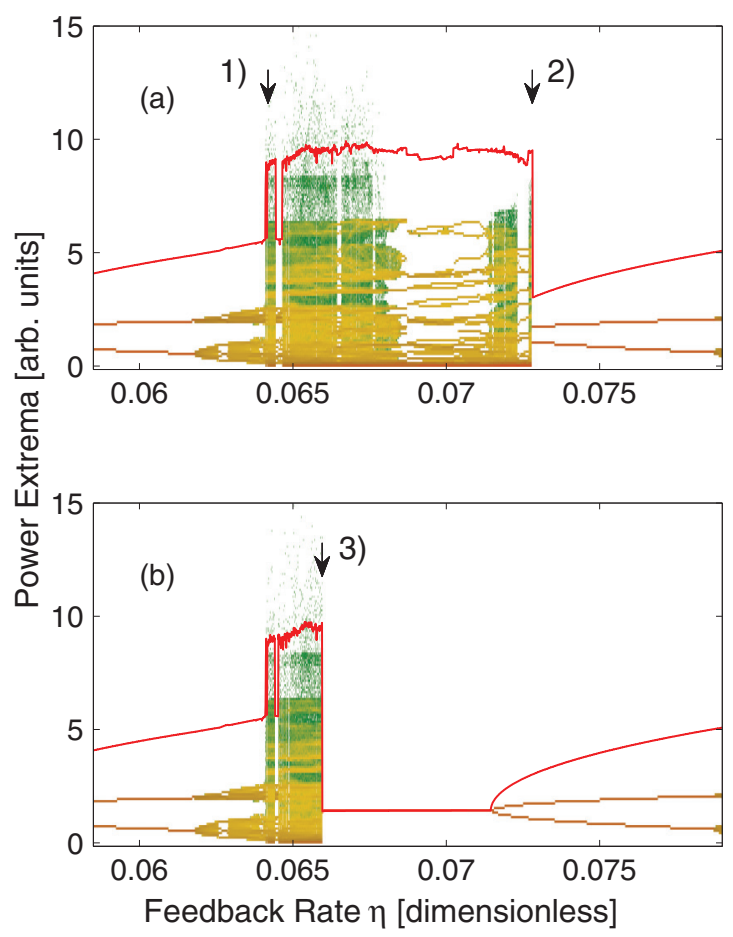

FIG. 1. (Color online) Bifurcation diagram when the feedback strength, $\eta$, increases (a) and then decreases (b). The pulse amplitude is plotted vs $\eta$, the top solid (red) line determines the threshold for a pulse to be considered an extreme event (equal to 5 times the standard deviation over the mean value). The color gray (browngreen) code on a logarithmic scale indicates the number of pulses. Lighter (brown) colors indicate highly probable pulse amplitudes; darker (green) colors, less probable amplitudes. The labels 1, 2, and 3 indicate the transitions discussed in the text. $\beta=0$; other parameters as indicated in the text.

reaching amplitudes higher than 10 , without a clear maximum. This abrupt change occurs at $\eta \sim 0.064$ and is referred to as transition 1. By further increasing the feedback strength, different dynamical regimes including periodic windows, chaos, and regular pulse packages (RPPs) occur [11-13].

At transition 1 the number of pulses (in color code) reveals that the pulse amplitudes are highly heterogeneous. There is a more densely visited range at low amplitudes that is similar before and after the expansion, while high-amplitude pulses occur only sporadically. The distribution of pulse amplitudes, presented in Fig. 2, has a long tail after the expansion (which reveals the existence of extreme values) [Fig. 2(b)] and a welldefined cutoff before the expansion [Fig. 2(a)].

In order to characterize the extreme pulses, they are defined quantitatively as those whose amplitude is higher than the mean value plus 5 times the standard deviation, $\sigma$, of the pulse amplitude distribution (the threshold is indicated by the red lines in Figs. 1 (top line) and 2 (vertical line)). As discussed in Sec. I, the criterion for defining quantitatively extreme pulses is quite arbitrary; however, the parameter region where extreme pulses are observed does not change significantly when the threshold is varied within the range of $5-8 \sigma$. We use $5 \sigma$ as a compromise solution to have good statistics without having to perform extremely long simulations (to observe a significant 

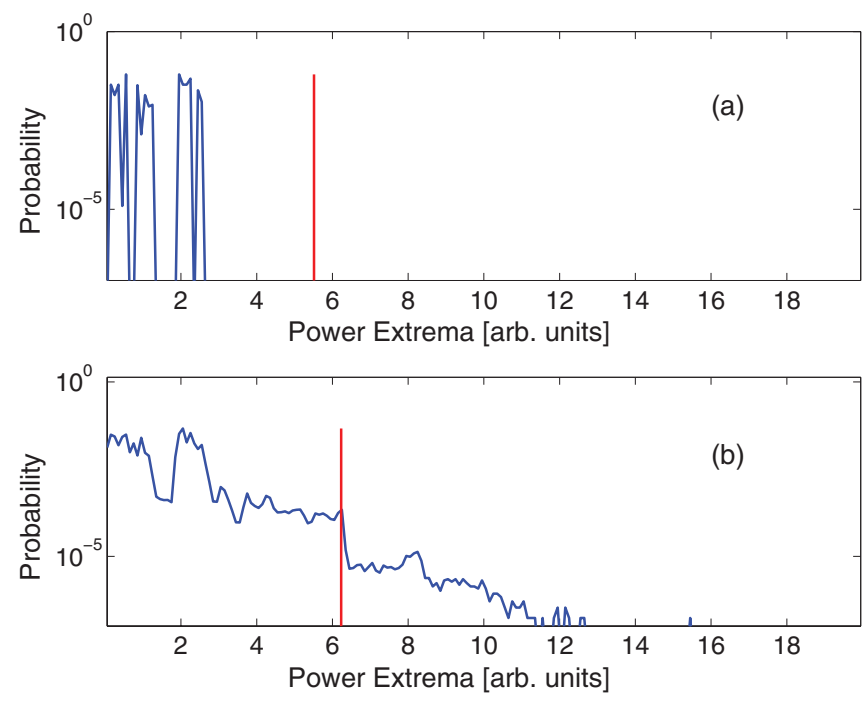

FIG. 2. (Color online) Distribution of pulse amplitudes for (a) $\eta=0.064$ and (b) $\eta=0.064095$, before and after the attractor expansion (transition 1), respectively. The vertical line indicates the threshold over which extreme pulses are defined, which is equal to the mean value pulse height plus 5 times the standard deviation of the pulse height distribution.

number of extreme pulses defined with a higher threshold would require much longer simulations).

Immediately after the expansion of the attractor (transition 1), deterministic intermittency occurs as shown in Fig. 3, where alternating intervals of high- and regular-intensity pulses are seen; during the intervals where the laser displays high pulses, only a few of these pulses are extreme, i.e., cross the threshold represented by the horizontal line.

A detail of an extreme pulse is shown in Fig. 4(a), and the projections of the trajectory in the planes (intensity, phase delay) and (carrier excess, phase delay) are shown in Figs. 4(b) and Fig. 4(c), respectively. Figure 4(c) also displays the position of the ECMs. As shown in this figure, high pulses

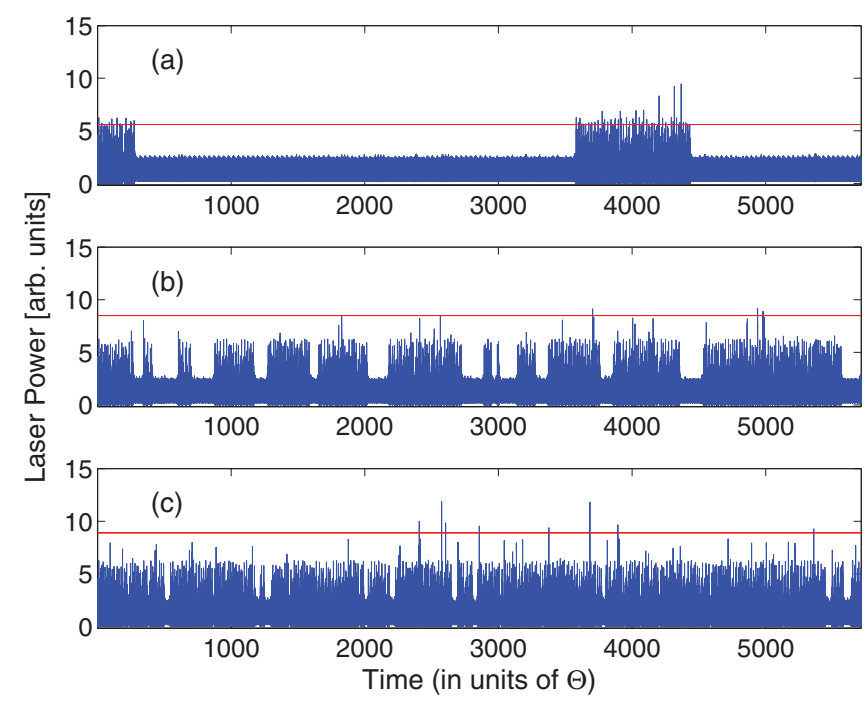

FIG. 3. (Color online) Deterministic intermittency after transition 1 ( $\beta=0$ ). (a) $\eta=0.064095$, (b) $\eta=0.06412$, and (c) $\eta=0.0642$.
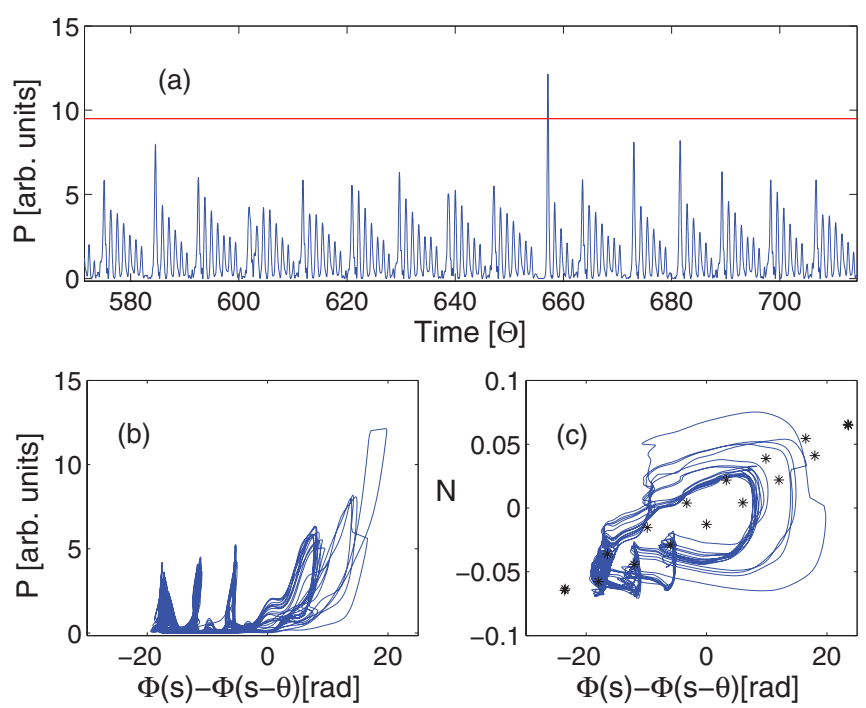

FIG. 4. (Color online) (a) Intensity time series where an extreme pulse is observed. (b, c) The phase portraits $[\Phi(s)-\Phi(s-\theta)], P]$ and $[\Phi(s)-\Phi(s-\theta), N] . \eta=0.066$; other parameters as in Fig. 1.

occur when the trajectory reaches large positive phase delays, $\Delta \phi_{s}$, while the regular-amplitude pulses do not approach that region of the phase space.

An abrupt expansion of pulse amplitudes similar to the one observed in transition 1 was reported in a semiconductor laser with cw optical injection [25]. In [25] the attractor expansion was interpreted as being due to the crossing of the attractor with a stable two-dimensional manifold of a saddle point and the subsequent convergence towards a narrow channel, i.e., a small region of the phase space (which the authors called a "narrow door") that triggers the extreme intensity pulses. Here, Figs. 5(a) and 6(a) show that there is a similar convergence of
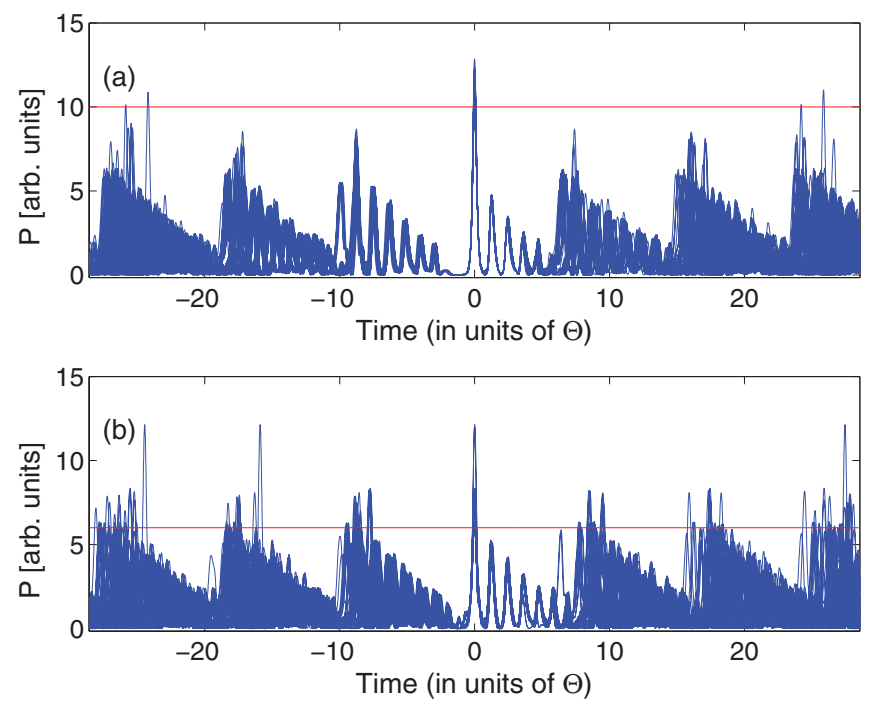

FIG. 5. (Color online) Superposition of extreme pulses, such that the time traces are centered at the peak of the pulse (see text for details). The feedback strength is $\eta=0.066$, and other parameters are as indicated in the text. The threshold for defining extreme pulses, indicated by the horizontal (red) line), is equal to 10 in (a) and is equal to 6 in (b). In both panels the number of pulses is 52 . 

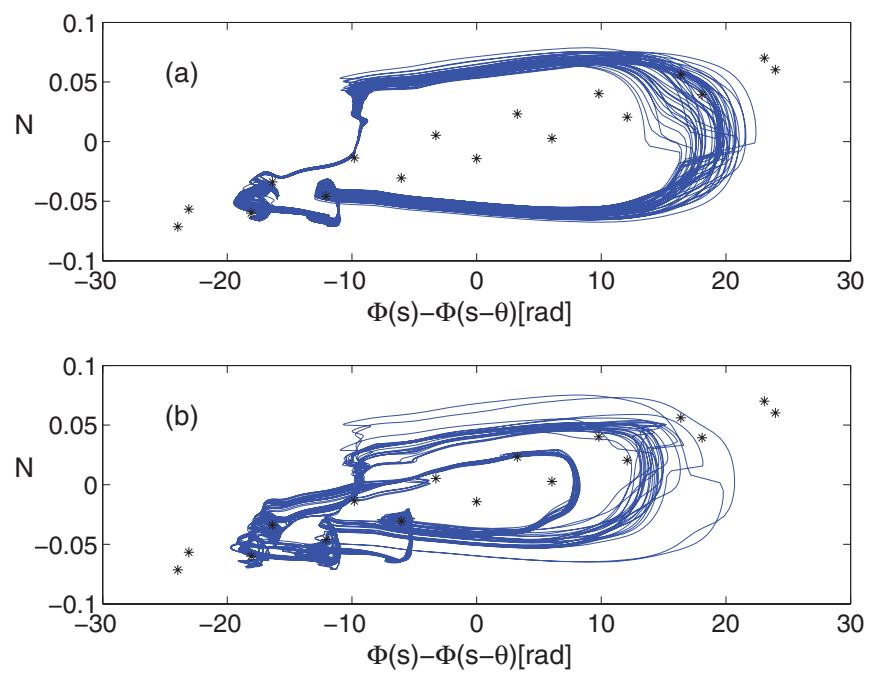

FIG. 6. (Color online) Phase portrait $[\Phi(s)-\Phi(s-\theta), N]$ displaying the superposition of the sections of the trajectory that contain the 52 pulses shown in Fig. 5. The amplitude threshold is equal to (a) 10 and (b) 6 .

the trajectories towards a narrow door before extreme intensity pulses occur.

Figure 5(a) was done by performing one long simulation of the noise-less rate equations, selecting the pulses that are above a certain amplitude threshold (in this case equal to 10 ) and superposing sections of the intensity time trace that contain the extreme pulse. Each section covers a time interval of $60 \mathrm{~ns}$ and the superposition is done by centering each section at the peak of the extreme pulse. One can observe that the superposition generates a narrow curve well before and after the extreme pulse occurs. This narrow curve is also shown in Fig. 6(a), which displays the superposition of sections of the trajectory (before and after the extreme pulse occurs) in the two-dimensional plane (phase delay, carrier density). On the contrary, in Figs. 5(b) and 6(b), which are done in the same way as Figs. 5(a) and 6(a), but with a lower threshold (now equal to 6), the superposition of sections of the intensity time trace containing a pulse above the threshold [Fig. 5(b)], and the superposition of the corresponding sections of the trajectory [Fig. 6(b)] is considerably more disperse and does not generate a narrow curve.

These plots support a qualitative comparison of the behavior with feedback and with injection and, despite the differences in both systems, suggests that extreme intensity pulses in the optical feedback case could occur through a similar mechanism as in the optical injection case. However, the feedback time delay renders the phase space infinite-dimensional, and thus, the topological analysis of the trajectories in phase space is difficult.

With strong feedback the attractor suddenly disappears [at $\eta \sim 0.073$; see Fig. 1(a)] and the intensity becomes oscillatory with constant low amplitude. We refer to this second abrupt change as transition 2. At this transition no sign of intermittency was observed. As shown in Fig. 7, before the attractor is destroyed the dynamics is periodic and corresponds to RPPs, which are well below the threshold of extreme pulses.
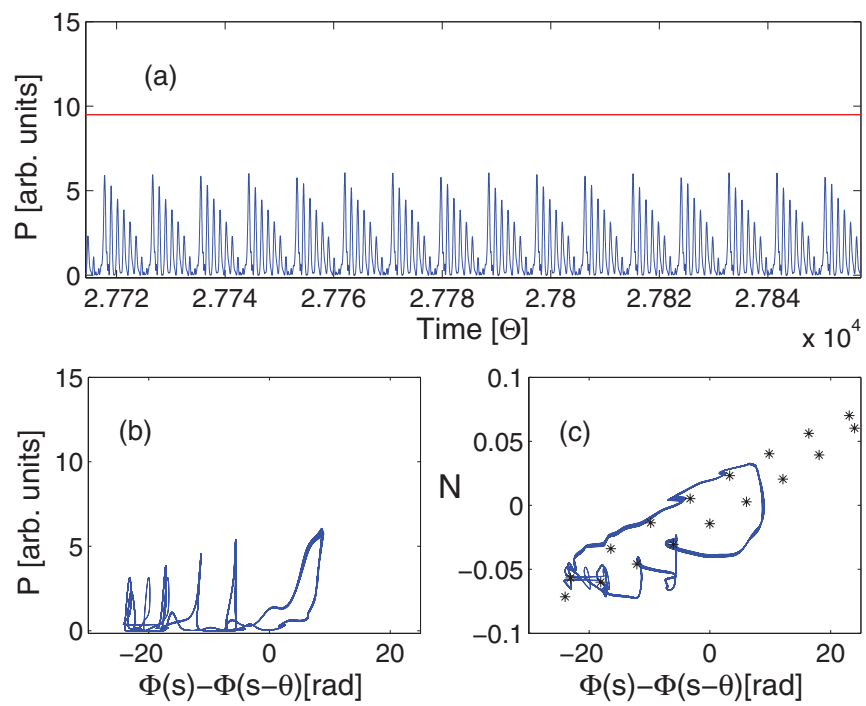

FIG. 7. (Color online) Time series of the laser intensity just before transition $2(\eta=0.073)$, displaying regular pulse packages. The horizontal line indicates the threshold for extreme pulses.

The new attractor is now followed by decreasing the feedback strength, and its bifurcations are shown in Fig. 1(b). The attractor described previously (referred to as attractor 1) coexists with this new attractor (referred to as attractor 2) over a wide range of feedback strengths, as could be expected for a dissipative system. The periodic intensity undergoes a Hopf bifurcation after which the $\mathrm{cw}$ regime is reached. Then the trajectory remains in this steady state (corresponding to a stable ECM) until the ECM disappears after a saddle-node

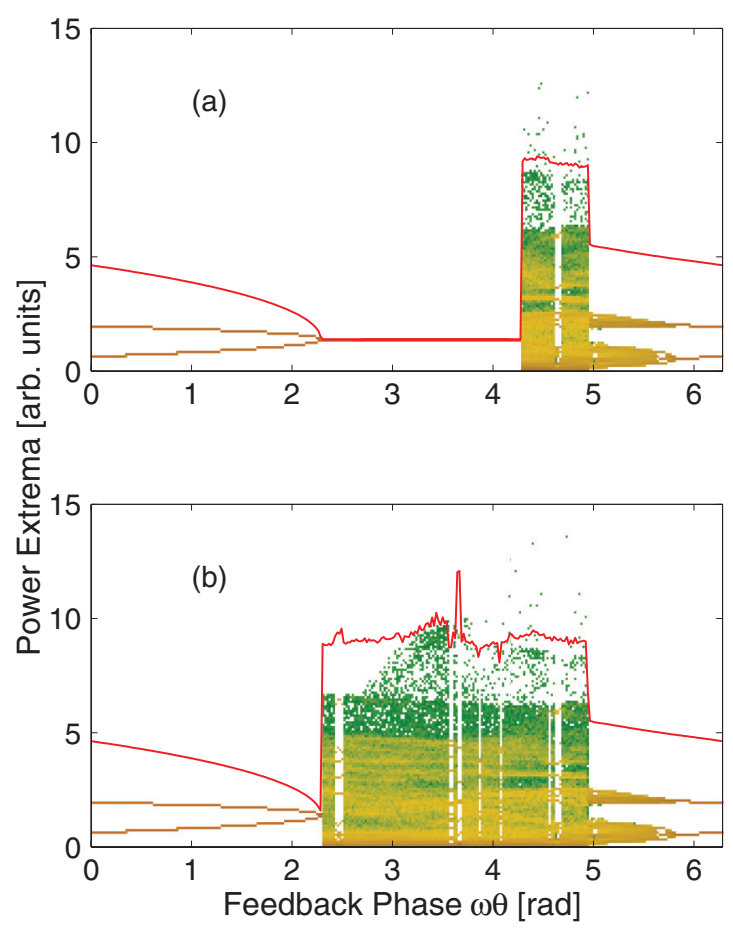

FIG. 8. (Color online) Bifurcation diagram when (a) increasing and (b) decreasing the feedback phase. $\eta=0.0642$; other parameters as in Fig. 1. 

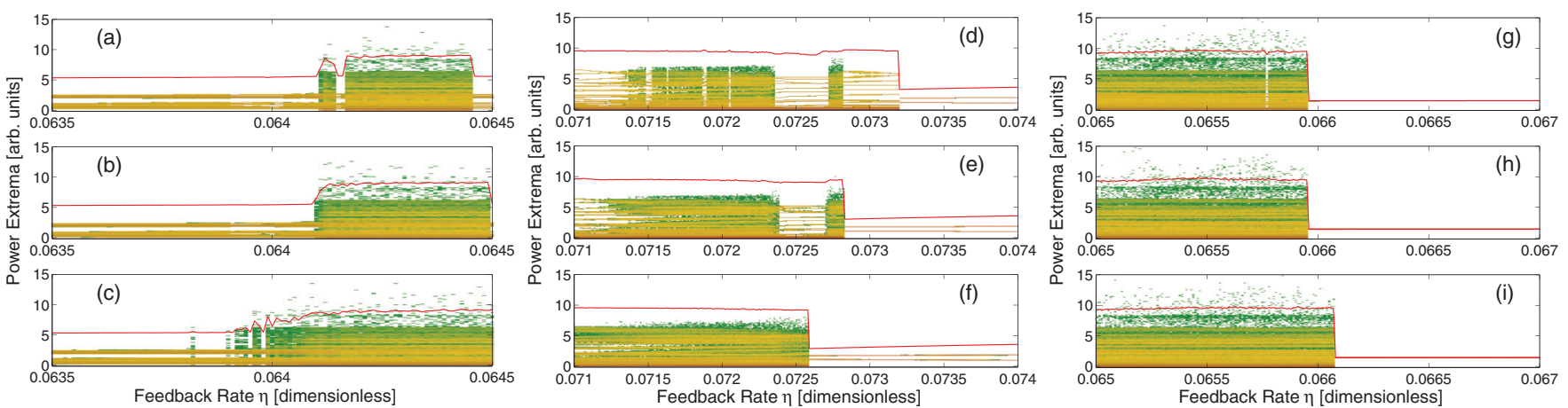

FIG. 9. (Color online) (a-c) Zoom of transition 1 when the feedback rate increases. (d-f) Zoom of transition 2 when the feedback rate increases. (g-i) Zoom of transition 3 when the feedback rate decreases. The noise strength is (a, d, g) $\beta=0,(\mathrm{~b}, \mathrm{e}, \mathrm{h}) \beta=10^{-4}$, and $(\mathrm{c}, \mathrm{f}, \mathrm{i})$ $\beta=10^{-3}$.

bifurcation occurring at $\eta \sim 0.066$. At this point, in the following referred to as transition 3 , the trajectory evolves back to attractor 1 , which sustains occasional extreme pulses, thus closing a hysteresis cycle. It seems that attractor 1 is not affected by the appearance (disappearance) [for increasing (decreasing) $\eta$ ] of the ECM. This could be due the fact that attractor 1 and the ECM are well separated in the phase space, because attractor 1 originates from a different ECM. Once the trajectory is back in attractor 1 , if we continue decreasing the feedback strength, the bifurcation scenario is the same as that observed when we increased the feedback [compare Fig. 1(b) after transition 3 with Fig. 1(a)]; i.e., no hysteresis is observed at transition 1.

The bifurcation scenario described for varying feedback strength is also observed when varying the feedback phase. Figure 8 shows three similar transitions when increasing and decreasing $w \theta$.

\section{B. Influence of spontaneous emission noise}

One could expect that random fluctuations, unavoidable in lasers, could induce switchings between the coexisting attractors previously discussed. In order to explore the effect of noise we did the bifurcation diagrams shown in Fig. 1, sweeping the feedback strength upwards and downwards, but now including additive noise in the simulations. In Fig. 9 we show in detail the three transitions with and without noise. Before transition 1 noise leads to occasional extreme intensity pulses [Figs. 9(a)-9(c)]. These noise-induced pulses are due to large excursions in the phase space, after which the trajectory relaxes back to the attractor.

The effect of noise observed at transitions 2 and 3 is the one expected when two attractors coexist: in Figs. 9(d)-9(f) (the feedback increases) one can note that noise anticipates the transition to attractor 2 , because transition 2 occurs at a smaller value of $\eta$. In Figs. 9(g)-9(i), we see that transition 3 (the feedback decreases) is also anticipated, i.e., occurs at a higher $\eta$ value.

\section{CONCLUSION AND DISCUSSION}

To summarize, we have studied numerically the dynamics of a semiconductor laser with optical feedback from a short external cavity and demonstrated the existence of parameter regions where the laser intensity displays extreme pulses. We have identified three relevant transitions involved in the appearance of the extreme pulses. Transition 1 and transition 2 are related to the appearance and destruction of the attractor that sustains extreme pulses. This attractor coexists with another smaller one over a broad range of feedback strengths (from transition 2 to transition 3 ) which defines a hysteresis cycle. We have also demonstrated that noise does not modify this scenario but only anticipates the different transitions.

While our results are fully consistent with the findings in Ref. [12], where the sequence of bifurcations leading to RPPs was studied in detail, our simulations suggest that RPPs and extreme pulses are different dynamical behaviors and occur in different parameter ranges. This is because the RPPs have a well-defined periodicity and pulse amplitudes that are not extreme (Fig. 7) and, also, because even when strong noise is included in the simulations, RPPs are robust and no extreme pulses are observed for parameters corresponding to RPP dynamics [Fig. 9(f)]. On the contrary, strong noise is capable of inducing extreme pulses for parameters before transition 1 (at transition 1 extreme pulses appear in the noiseless simulations) [Fig. 9(c)].

Since diode lasers with integrated short external cavities are nowadays widely used in many applications that require singlefrequency, compact, and efficient light sources, our results can help in avoiding extreme intensity pulses in these devices, which might originate due to uncontrolled small variations in the feedback parameters.

\section{ACKNOWLEDGMENTS}

We would like to thank Manuel Matías and Ulrike Feudel for interesting and inspiring discussions. This work was supported in part by Grant No. FA8655-12-1-2140 from EOARD US, Grant No. FIS2012-37655-C02-01 from the Spanish MCI, and Grant No. 2009 SGR 1168 from the Generalitat de Catalunya. C. Masoller acknowledges support from the ICREA Academia programe. J.Z.M. acknowledges support from FISICOS Grant No. FIS2007-60327 of the Spanish MCI and INTENSE@COSYP Grant No. FIS2012-30634 of the FEDER. J.A.R. acknowledges support from Grant No. BES-2008-003398 and thanks the UPC for hospitality during his visit, during which part of this work was done. 
[1] J. Masoliver, M. Montero, and J. Perelló, Phys. Rev. E 71 , 056130 (2005).

[2] C. Nicolis and G. Nicolis, Phys. Rev. E 85, 056217 (2012).

[3] T. Schweigler and J. Davidsen, Phys. Rev. E 84, 016202 (2011).

[4] R. W. Katz, Climatic Change 100, 71 (2010).

[5] V. I. Yukalov, E. P. Yukalova, and D. Sornette, Eur. Phys. J. Special Topics 205, 313 (2012).

[6] H. E. Roman, R. A. Siliprandi, C. Dose, and M. Porto, Phys. Rev. E 80, 036114 (2009).

[7] M. G. Kovalsky, A. A. Hnilo, and J. R. Tredicce, Opt. Lett. 36, 4449 (2011).

[8] C. Bonatto, M. Feyereisen, S. Barland, M. Giudici, C. Masoller, J. R. Rios Leite, and J. R. Tredicce, Phys. Rev. Lett. 107, 053901 (2011).

[9] K. Schires, A. Hurtado, I. D. Henning, and M. J. Adams, Electron. Lett. 48, 14 (2012).

[10] A. K. Dal Bosco, D. Wolfersberger, and M. Sciamanna, Opt. Lett. 38, 703 (2013).

[11] T. Heil, I. Fischer, W. Elsäßer, and A. Gavrielides, Phys. Rev. Lett. 87, 243901 (2001).

[12] A. Tabaka, K. Panajotov, I. Veretennicoff, and M. Sciamanna, Phys. Rev E 70, 036211 (2004).

[13] A. Tabaka, M. Peil, M. Sciamanna, I. Fischer, W. Elsäßer, H. Thienpont, I. Veretennicoff, and K. Panajotov, Phys. Rev. A 73, 013810 (2006).
[14] R. Lang and K. Kobayashi, IEEE J. Quantum Electron. 16, 347 (1980).

[15] D. M. Kane and K. A. Shore, Unlocking Dynamical Diversity: Optical Feedback Effects on Semiconductor Lasers (Wiley, New York, 2005).

[16] J. Ohtsubo, Semiconductor Lasers: Stability, Instability and Chaos, 2nd ed. (Springer, Berlin, 2010).

[17] C. Kharif, E. Pelinovsky, and A. Slunyaev, Rogue Waves in the Ocean (Springer, Heidelberg, 2009).

[18] S. Albeverio, V. Jentsch, and H. Kantz, Extreme Events in Nature and Society, The Frontiers Collection (Springer, Heidelberg, 2006).

[19] T. Sano, Phys. Rev. A 50, 2719 (1994).

[20] C. Masoller and N. B. Abraham, Phys. Rev. A 57, 1313 (1998).

[21] R. L. Davidchack, Y.-C. Lai, A. Gavrielides, and V. Kovanis, Physica D 145, 130 (2000).

[22] A. Torcini, S. Barland, G. Giacomelli, and F. Marin, Phys. Rev. A 74, 063801 (2006).

[23] J. Zamora-Munt, C. Masoller, and J. Garcia-Ojalvo, Phys. Rev. A 81, 033820 (2010).

[24] K. Al-Naimee, F. Marino, M. Ciszak, S. F. Abdalah, R. Meucci, and F. T. Arecchi, Eur Phys. J. D 58, 187 (2010).

[25] J. Zamora-Munt, B. Garbin, S. Barland, M. Giudici, J. R. Rios Leite, C. Masoller, and J. R. Tredicce, Phys. Rev. A 87, 035802 (2013). 